\title{
Analysis of fuel particle size based on particle swarm fuzzy C-average clustering
}

\author{
WAN Li ${ }^{1, a}$, MA Yongjun ${ }^{1, b}$, GUO Jianmin ${ }^{2, c}$ \\ ${ }^{1}$ Tianjin University of Science \&Technology, Tianjin, China \\ ${ }^{2}$ Tianjin Polytechnic University, Tianjin, China \\ awanli@mail.tust.cn, byjma@tust.edu.cn, coguojianmin@tjpu.edu.cn
}

\begin{abstract}
Keywords: Particles; The particle swarm fuzzy C - average clustering; Distribution; morphology
\end{abstract}

\begin{abstract}
Fuel particle size and distribution is the key to energy saving of blast furnace. The fuel particle size will have influence to the highest temperature of the sintered material layer. The paper describe a method of adhesive particle image segmentation using based on particle swarm optimization fuzzy C -means clustering. It can measure particle size of fuel using OPENCV image processing real-time. Some experimental results are given, which show that the algorithm has the effective ability of searching global optimal solution. It has get very good effect on particle statistics.
\end{abstract}

\section{Introduction}

The fuel is the foundation of high temperature furnaces stable operation, so the need of original fuel quality is high[1]. In order to guarantee the stability of the original fuel quality, strengthening digital management is essential. The important operation of furnaces temperature is to ensure the ventilation of fuel columns in the furnaces. In addition, one of the important factor which can affect the ventilation is the particle size and the distributed composition of the fuel those are sent into the furnaces. When the size of the fuel is too big, the decomposition is slow and consumption rate is high, the fuel will burn out after arriving the combustion zone. When the size of is too small, the ventilation in the furnace will be affected and form over fire.

Blast furnace iron making is based on concentrate, the particle size of fuel should be uniform. The size of fuel between $5-15 \mathrm{~mm}$ should be less than $30 \%$ of the total, the size smaller than $5 \mathrm{~mm}$ should be less than $5 \%$ of the total. The ingredient and the quality must be stable, the harmful substances should be few[2].

The main methods for particle size detection currently contains mechanical mesh sieve method, laser diffraction, electrical induction method etc.[3]. With the application of digital image processing technology in the recognition particle size area, particle measuring technology own the advantages of wide using range, accuracy measurement and high precision etc.. Although there are lots of methods for particle size detection nowadays, most of them exists the problems of long period, complicated steps, and low accuracy rate. Particle size detection is the important quality standard, a good measure method and the accuracy measure result play an important role in the fuel using and the processing applications[4].

With the rapidly development of the computer, CPU processing speed is greatly improved, CCD technology is becoming stronger. These factors make the quality of digital images higher, the cost of equipment on the image decrease, and make the image technology using into mineral process possible[5]. As a result, the image processing technology can be applied into varied kinds of processing and manufacturing industry.

According the features of the fuel particle image, the text analyze the performance of particle and the applicability of using in the particle image. In the text, the methods include points fuel particle swarm fuzzy C- average clustering algorithm for image segmentation, colleting the perimeter, area, particle size, shape index and shape feature parameters.

The study applies Open CV library and 32 bites $\mathrm{C} / \mathrm{C}++$ translator-editor system. Under the 
VC++6.0 circumstance, the automatic segmentation, edge tracking, testing and statistical analysis of the particle have been realized. In the paper, statistics of computing and distribution of particle size real time have also been completed, and according the statistics, correct and suitable method for quantitative analysis of particle size has been offered[6].

\section{Particle size analyze system}

The analyze system of particle size consists of hardware and software two groups. The original fuel size on-line automatic detection device includes camera and protection devices, external light source, hood, industrial computers, and fiber optic cable as major components[7], as the figure 1 shown. It mainly accomplish the work of particle image acquisition, storage and analysis.

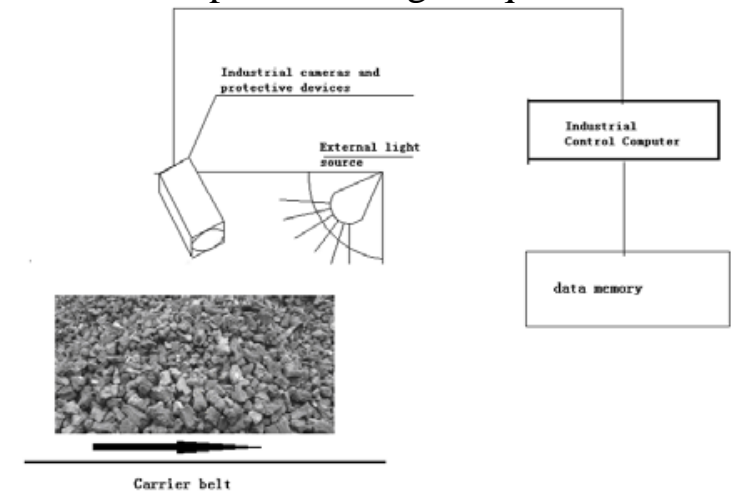

Fig.1 Automatic detection device of fuel particle on-line

Industrial cameras used a fixed focal length of high-speed industrial camera, the installed camera was perpendicular to shoot material, as the production environment of blast furnaces was filled with dust, the protector for camera was essential, in addition, the purge with compressed air could be used in order to keep the camera lens clear. As a result, the image of the material on the conveyor could be shot clear, and the images would be sent to the industry computer. And then the computer computed the size of the original fuel out, the computer got a picture every 10s, the result was saved in the memory.

\section{Particle detection algorithm principle}

The algorithm principle is shown in figure 2. The camera which was used in the experiment was camera DS-2CD4032FWD-SDI. It is 300 million pixels CMOS ICR Day \& Night Gun Digital Camera, with H.264 High Profile code, the highest resolution can be up to 300 million pixels @20fps and exports 1080p@30fps real time image.

The method for dealing with the images was median filtering method. The median filtering is one of non-linear signal processing techniques which can effectively suppress noise and it is based on sort statistical theory. The basic principle of median filtering is to replace a point in the digital image or digital sequence with the median of the each point in the neighborhood. The aim is to make the pixel value be closest to the real value and remove the single noise point. The method adopted dimensional slide template of a certain structure, and pixel in the template would be sorted by size according the pixel value. Dimensional data sequences those were monotonically rising or falling came into being. They were usually $3 * 3,5 * 5$ area or different shapes. The study adopter the $3 * 3$ square templates.

When the median filtering eliminates noise, the mainly two factors which are related but entirely separated the median filtering dependents on are the spatial extent of the neighborhood and number of pixels involved. Figure 3 is the result of comparing before and after treatment with median filtering. After the treatment, the edge of collected particle is clear and inner grayscale difference becomes smaller. 


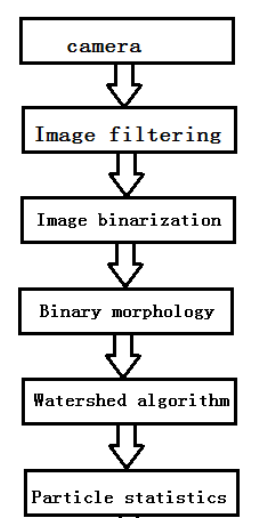

Fig.2 The principle diagram of particle detection

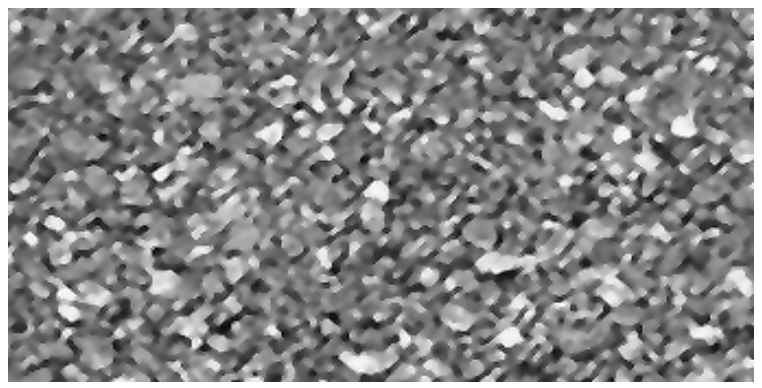

Fig.3 (a) Particle image

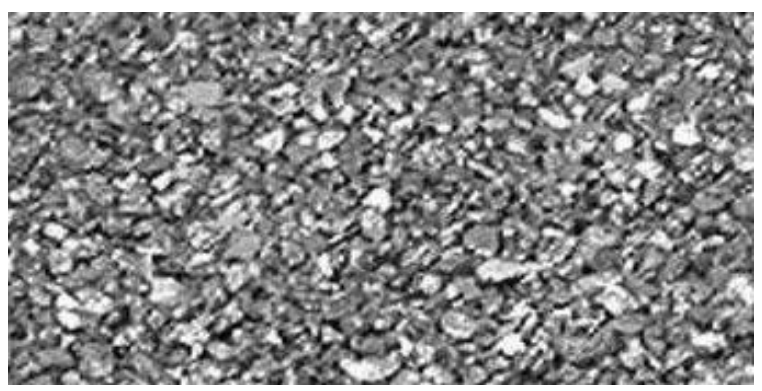

Fig.3 (b) Medium value filter processing image

Fig.3 Medium value filter processing

Because of the different between the background and the aim area, the binary image after segmentation shows discrete noise more or less. When high-frequency ingredients of binary image are too much, the performance figure is that the edge shows zigzag shape. Morphological filtering not only can remove the discrete pitting noise but also makes the edge became even and smooth. Additional, high light spots in the particle easily form empty hole after binarization and the image got is not good for feature extraction. Seed filling will solve the problem effectively and guarantee the effectiveness of the size measure meanwhile.

For the measure of powder particle, the grayscale images got are usually shown with adhesions, even with some spots overlapping. The important to obtain the parameters such as size and number is the separation adhesions of particle and make them independent. Watershed segmentation is based on the pixel classification method, and it can solve the problem of the particle adhesions segmentation[8].

Watershed algorithm is based on the image segmentation, the result of watershed exchange is collection basin image of the image input. The edge point between collection basins is the watershed. Obviously, what the watershed shows is the maxima point of the input image. To get the edge information of the image, the gradient image usually is used as the input image.

$$
\operatorname{grad}(f(x, y))=\sqrt{[f(x, y)-f(x-1, y)]^{2}+[f(x, y)-f(x, y-1)]^{2}}
$$

$f(x, y)$ is the original image, $\operatorname{grad}($.$) is the gradient computation.$

Watershed algorithm has a good response to faint edge, the noise and tiny grayscale change on the surface of the object in the image will cause over segmentation. But the good response comes 
from the guarantee of closed and continuous edge. Closed collection basins those were got from watershed algorithm make the possibility for analysis of area feature[9].

The method of region merging can improve the situation of over segmentation. According a certain principle, regions adjacent or close are merged constantly. Fuzzy C- average clustering is an effective method for change the over segmentation phenomenon of watershed algorithm.

The mainly thinking of fuzzy C-average clustering algorithm is that dividing dataset $X=\left\{x_{1}, x_{2}, \ldots, x_{n}\right\} \in R_{p n}$ into C kinds, $\mathrm{Xk}$ is the any sample in $\mathrm{X}$ and the membership of $\mathrm{Xk}$ to I is uik. One fuzzy membership matrix $U=\left\{u_{i k}\right\} \in R_{p n}$ can represent the result[10] and satisfies:

$$
\left\{\begin{array}{l}
u_{i k} \in[0,1], \forall i, k \\
0<\sum_{k} u_{i j}<n, \forall i \\
\sum_{i} u_{i j}=1, \forall k
\end{array}\right.
$$

Fuzzy C-average clustering is realized through minimizing the objective functions $J_{m}(U, V)$ of membership matrix $\mathrm{U}$ and cluster center $\mathrm{V}$.

$$
J_{m}(U, V)=\sum_{k=1}^{n} \sum_{i=1}^{c}\left(u_{i k}\right)^{m} d_{i k}^{2}\left(x_{k}, v_{i}\right)
$$

In the formula, $\mathrm{U}=\left\{\boldsymbol{u}_{i k}\right\}$ is the membership matrix which is satisfied formula (3), $V=\left\{v_{1}, v_{2}, \ldots, v_{c}\right\} \in R_{p c}$ is the center point set of C clustering, $m \in[1, \infty]$ is weighted index, $d_{i k}^{2}\left(x_{k}, v_{i}\right)$ is the distance from the $\mathrm{k}$-th sample to the center of I-th kind. The defining equation is

$$
d_{i k}^{2}\left(x_{k}, v_{i}\right)=\left|x_{k}-v_{i}\right|_{A}^{2}
$$

A is $\mathrm{p}^{*} \mathrm{p}$ positive definite matrix.

When fuzzy C-average clustering algorithm is used to image segmentation, the fast FCM algorithm which is based on grayscale histogram is adopted. The objective function was defined as:

$$
W_{m}(U, V)=\sum_{k=0}^{L} \sum_{i=1}^{c}\left(u_{i k}\right)^{m}\left(d_{i k}\right)^{2} h(k)
$$

$h(k)$ is grayscale histogram of image, $\mathrm{k}$ is the grayscale, $k=0 \sim L$, $\mathrm{L}$ is the maximum of grayscale. $u_{i k}$ is the fuzzy membership of k-th grayscale in the i-th kind. dik is the distance from the k-th greyscale to the clustering center of the $\mathrm{i}$-th, the define is:

$$
\left(d_{i k}\right)^{2}=\left\|k-z_{i}\right\|^{2}
$$

$\mathrm{Zi}$ is the grayscale value of the $\mathrm{i}$-th clustering center.

To evaluate each spot in the particle group in the particle group fuzzy C-average clustering algorithm and defining the fitness function.

$$
f\left(x_{i}\right)=\frac{k}{J_{m}(W, Z)}
$$

$\mathrm{k}$ is constant, is the total of sum of between-class scatter.

If the kind numbers $\mathrm{C}$, fuzzy index $\mathrm{m}$, group scale $\mathrm{N}$, learning factor $\mathrm{c} 1$ and $\mathrm{c} 2$, inertia weight $\mathrm{w}$ are certain, clustering center is:

$$
z_{i}=\frac{1}{\sum_{k=0}^{L}\left(u_{i k}\right)^{m}} \sum_{k=0}^{L}\left(u_{i k}\right)^{m} h(k)
$$

Every clustering center computes the membership uij:

$$
\mathrm{i}=1,2 \ldots \mathrm{n}, \mathrm{j}=1,2 \ldots \mathrm{c} \quad u_{i, j}=\frac{1}{\sum_{t=1}^{c}\left(\frac{d_{i j}(k)}{d_{i t}(\mathrm{k})}\right)^{\frac{2}{m-1}}}
$$

\section{Experimental results}

The software environment is $\mathrm{VC}++6.0$ and Open $\mathrm{CV}$, the constant of clustering algorithm $\mathrm{m}=2$, 
the maximum number of iterations is 500. In the experiment, watershed and text algorithm were used to process images. Figure 4 shows the result of segmenting image with watershed algorithm and drawing edges with Canny algorithm. Figure 5(a) shows the result of segmenting images with particle group fuzzy C-average clustering algorithm. It is obviously that the algorithm in the study splitting the objective and background effectively and getting a better recognition result.

OpenCV offered the language interfaces such as Python、Ruby、MATLAB and realized images processing and the common algorithms on visual aspects. Function cvContourArea computed the area of the whole and a part of the contour. The OpenCV finished the size computation and the statistic of fuel particle.

Figure 5(b) is the statistical figure of the fuel particle which is used to analyze the particle distribution of the fuel.

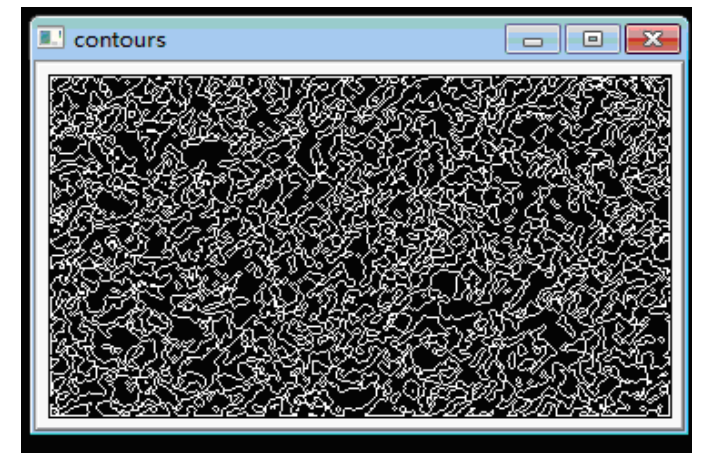

Fig.4 Edge image the traditional watershed algorithm

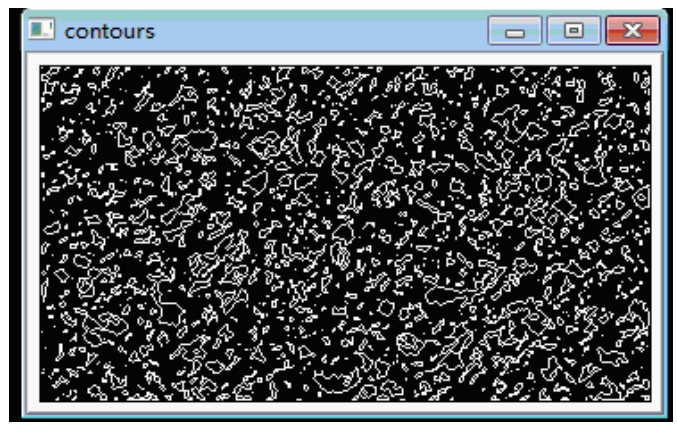

Fig.5 (a) Image edge of particle swarm fuzzy C - average clustering algorithm

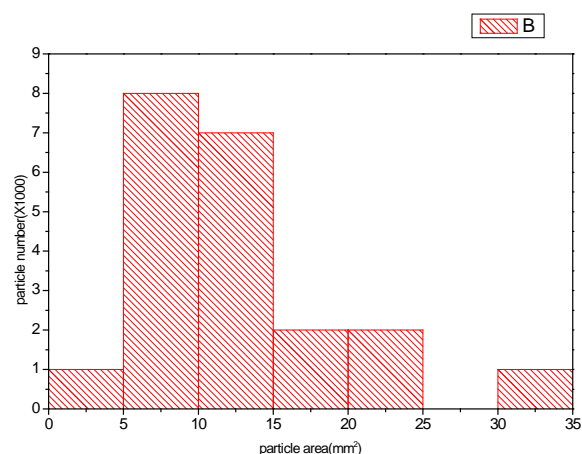

Fig. 5(b) The statistical figure of the fuel particle

\section{Conclusion}

The size of particles is the most basic constant of the fuel. The text is based on image analysis theory. The requirement of measuring and analysis particle size can be met by the particle analysis system which is developed in the study.

The image segmentation algorithm which is based on particle groups fuzzy C-average clustering has been got an ideal result in the particle analysis. The experiment shows that the algorithm can solve the problem of traditional FCM[11] getting into minimum value, with stronger capability of global search and quicker speed on convergence. 


\section{References}

[1] Liu Zhulin, Wang Jianli, Su Zhenjiang1, Yang Xu. Analysis of the measurement on reducing the fuel rate of blast furnace in pingxiang iron and steel Co.Journal of Hunan University of Technology, 2012, 26(1):96-100.

[2] The quality requirements and impact to raw fuel in blast furnaces iron making. 2012-09-05. http://jiaotan.mysteel.com/12/0905/09/1284BFD02B6AA5DA.html

[3] WEN Haibin, JIA Ruiqiang. The application of computer image processing technology to on-line grain size measurement in mineral processing [J]. China Molybdenum Industry, 2005, 29(1):22-25.

[4] HU Song- qing, LI Lin, G UO Si- yuan. CAI Miao- yanModern technology of particle size measurement. Modern Chemical Industry. Jan. 2002. 22(1).

[5] Wang Wei, The development of blast furnaces combustion monitoring system. Research on Iron \& Steel. January 1997. No.1.

[6] Zhang Yu, Xu Ruiquan. Improvement on watershed algorithm of OpenCV and its application in cell image segmentation. Journal of Computer Applications. 2012, 32(SI):134-136.

[7] WANG Xunfu. Research on on-line detecting technology of material and fuel size and distribution on baosteel blast furnace[C]. 2013-the fifth baosteel academic annual meeting.

[8] ZHANG Shuo, YANG Yaoquan, MIAO Chunyan, HUN Shenghui. Image segmentation about the touched object based on improved method of watershed algorithm [J].Journal of Image and Signal Processing, 2013, 2, 33-36

[9] DIAO Zhi-hua, ZHAO Chun-jiang, GUO Xin-yu, LU Sheng-lian, WANG Xiu-hui. Research on Improved Methods of Watershed Algorithm. Computer Engineering. September 2010.Vol.36. No.17.

[10] PU Peng-bo, WANG Ge, LIU Tai-an. Research of improved fuzzy C-means algorithm based on particle swarm optimization. Computer Engineering and Design.Aug.2008.26(16).

[11] LI Lili,LI Ming,LIU Xiyu.Image segmentation algorithm based on particle swarm optimization fuzzy C-means clustering[J].Computer Engineering and Applications, 2009,45(31):158-160. 